\title{
Small Limit Points of Mahler's Measure
}

\author{
David W. Boyd and Michael J. Mossinghoff
}

\section{CONTENTS}

\section{Introduction}

2. Hexanomials with Small Measure

3. Computing Measures of Two-Variable Polynomials

4. Searching for Small Measures

5. Limit Points Less than $\mathbf{1 . 3 7}$

6. True Limit Points

7. Explicit Formulas

Acknowledgments

References

2000 AMS Subject Classification: Primary 11C08; Secondary 11R09, 11Y35

Keywords: Lehmer's problem, Mahler measure
Let $M\left(P\left(z_{1}, \ldots, z_{n}\right)\right)$ denote Mahler's measure of the polynomial $P\left(z_{1}, \ldots, z_{n}\right)$. Measures of polynomials in $n$ variables arise naturally as limiting values of measures of polynomials in fewer variables. We describe several methods for searching for polynomials in two variables with integer coefficients having small measure, demonstrate effective methods for computing these measures, and identify 48 polynomials $P(x, y)$ with integer coefficients, irreducible over $\mathbb{Q}$, for which $1<M(P(x, y))<1.37$.

\section{INTRODUCTION}

Given a Laurent polynomial $P\left(z_{1}, \ldots, z_{n}\right)$ with integer coefficients, its Mahler's measure $M(P)$ is defined as the geometric mean of $|P|$ over the real $n$-torus $\mathbb{T}^{n}$,

$$
\begin{aligned}
& M(P)= \\
& \exp \left(\int_{0}^{1} \ldots \int_{0}^{1} \log \left|P\left(e\left(t_{1}\right), \ldots, e\left(t_{n}\right)\right)\right| d t_{1} \ldots d t_{n}\right),
\end{aligned}
$$

and here $e(t)$ as usual denotes $\exp (2 \pi i t)$. We also define the logarithmic Mahler's measure of $P$, denoted $m(P)$, by

$$
m(P)=\log M(P) .
$$

For a polynomial in one variable, one may obtain a more elementary expression for the measure by using Jensen's formula. Writing $P(x)=a_{0} \prod_{j=1}^{d}\left(x-\alpha_{j}\right)$, we have

$$
M(P(x))=\left|a_{0}\right| \prod_{j=1}^{d} \max \left(1,\left|\alpha_{j}\right|\right) .
$$

Note that (1-2) also follows from the observation that the measure is multiplicative, combined with the special case

$$
M(a x+b)=\max (|a|,|b|),
$$

which is easy to establish by elementary means.

Certainly $M(P(x))=M(P(-x))$, and using (1-1) or $(1-2)$ it is easy to show that $M(P(x))=M\left(P^{*}(x)\right)$, where $P^{*}(x)=x^{\operatorname{deg}(P)} P(1 / x)$ is the reciprocal polynomial of $P(x)$. More generally, an important and useful 
property of Mahler's measure for polynomials in several variables is its invariance under a large class of variable substitutions. Let $\mathbf{z}=\left(z_{1}, \ldots, z_{n}\right)$, and for a matrix $A=\left(a_{j, k}\right) \in \mathrm{GL}(n, \mathbb{Z})$, let $\pm \mathbf{z}^{A}$ denote any of the $2^{n}$ $n$-tuples of the form

$$
\left( \pm \prod_{j=1}^{n} z_{j}^{a_{1, j}}, \ldots, \pm \prod_{j=1}^{n} z_{j}^{a_{n, j}}\right) .
$$

Then

$$
M(P(\mathbf{z}))=M\left(P\left( \pm \mathbf{z}^{A}\right)\right) .
$$

See [Schinzel 00, page 226] for a proof.

In this paper, we investigate some questions regarding the spectrum of values of Mahler's measure; in particular, we are interested in especially small values of the measure. Let $L$ denote the set of values of Mahler's measure of univariate (Laurent) polynomials with integer coefficients,

$$
L=\{M(P): P \in \mathbb{Z}[x]\},
$$

and let $\mathbb{L}$ denote the set of values of measures of polynomials in any number of variables,

$$
\mathbb{L}=\bigcup_{n \geq 1}\left\{M(P): P \in \mathbb{Z}\left[z_{1}, \ldots, z_{n}\right]\right\},
$$

so $L \subseteq \mathbb{L}$.

For one-variable polynomials, clearly the minimal value of $L$ is 1 , and a classical result of Kronecker implies that $M(P(x))=1$ precisely when $P(x)$ is a product of cyclotomic polynomials and a monomial of the form $x^{m}$. Rather little is known, however, about values in $L$ near 1. In 1933, D. H. Lehmer [Lehmer 33] found that the polynomial

$$
x^{10}+x^{9}-x^{7}-x^{6}-x^{5}-x^{4}-x^{3}+x+1
$$

has Mahler's measure $\lambda_{0}=1.176280 \ldots$, and he asked if there exist any smaller values in $L$ exceeding 1 . This question of determining whether 1 is a limit point of $L$ is known as Lehmer's problem.

Despite several extensive searches [Boyd 80, Boyd 89, Mossinghoff 98, Mossinghoff et al. 98, Rhin and SacÉpée 03, Flammang et al. 06], the value $\lambda_{0}$ remains the second-smallest known value of $L$. Lehmer's problem has, however, been solved in some special cases. In 1951, Breusch [Breusch 51] proved that if $P(x) \in \mathbb{Z}[x]$ satisfies $P(0) \neq 0$ and $p \neq \pm P^{*}$, then $M(P(x)) \geq$ $M\left(X^{3}-x^{2}-1 / 4\right)=1.179652 \ldots$. This was sharpened by Smyth in 1971 [Smyth 71] to $M(P(x)) \geq M\left(x^{3}-x-1\right)=$ $1.324717 \ldots$. This value, which we denote $\theta_{0}$, was in fact shown by Siegel to be the smallest Pisot number [Siegel 44]; the value $\lambda_{0}$ is the smallest known Salem number (see for instance [Boyd 77]).

Multivariable analogues of Kronecker's result and Smyth's theorem are established in [Boyd 81a]. There it is shown that if $P(\mathbf{z})$ is an irreducible polynomial in $n$ variables with integer coefficients having $M(P(\mathbf{z}))=1$, then $P(\mathbf{z})=\Phi\left(z_{1}^{a_{1}} \cdots z_{n}^{a_{n}}\right)$, for some cyclotomic polynomial $\Phi(x)$ and integers $a_{1}, \ldots, a_{n}$ (see also [Schinzel 00, page 260]). Further, it is established that values smaller than $\theta_{0}$ in $\mathbb{L}$ may be produced only by reciprocal multivariable polynomials: those for which $P(\mathbf{z}) / P\left(\mathbf{z}^{-1}\right)$ is a unit times a monomial in $z_{1}, \ldots, z_{n}$.

The spectrum of values of $\mathbb{L}$ is also connected to the study of Lehmer's problem, due to the important fact that measures of polynomials in $n$ variables are limiting values of measures of polynomials in fewer variables. Specifically, if $\mathbf{r}=\left(r_{1}, \ldots, r_{n}\right)$ is a vector of integers and if $\mu(\mathbf{r})$ denotes the quantity

$$
\mu(\mathbf{r})=\min \left\{\|\mathbf{s}\|: \mathbf{s} \in \mathbb{Z}^{n} \text { and } \mathbf{r} \cdot \mathbf{s}=0\right\},
$$

then

$$
\lim _{\mu(\mathbf{r}) \rightarrow \infty} M\left(P\left(x^{r_{1}}, \ldots, x^{r_{n}}\right)\right)=M(P(\mathbf{z})) .
$$

This was conjectured in [Boyd 81b] and proved in [Lawton 83] (see also [Schinzel 00, page 237]). It was also noted in [Boyd 81b] that a solution of Lehmer's problem would follow if one could show that every limit point of $L$ is an element of $\mathbb{L}$, that is, if $\mathbb{L}$ is closed. We review the proof here: suppose $\mathbb{L}$ is closed and that 1 is a limit point of $L$. Because the measure is multiplicative, it follows that $\mathbb{L}=[1, \infty)$, but this is impossible because $\mathbb{L}$ is a countable set.

It was suggested in [Boyd 81b] that the smallest limit points of $L$ would be given by measures of two-variable polynomials, and candidates were proposed for the smallest two limit points, as well as a third value less than $\theta_{0}$. A fourth measure smaller than $\theta_{0}$ was exhibited in [Mossinghoff 98]. It appears however that no systematic search for other small limit points has been performed.

In this article, we search for additional small limit points of $L$ realized by measures of two-variable polynomials and identify 48 limit points less than 1.37. We need to consider only irreducible $P(x, y) \in \mathbb{Z}[x, y]$ with $M(P)>1$, and we need only consider one representative from each class of polynomials related by (1-4). In particular, we seek only primitive polynomials, that is, those not of the form $P\left( \pm \mathbf{z}^{A}\right)$ for $A \in \mathrm{GL}(n, \mathbb{Z})$ with $|\operatorname{det}(A)|>1$. For example, we accept $P(x, y)=1+x+y$, but not $1+x^{2}+y^{3}$ or $1-x y^{2}+x^{2} y$. 
We remark that from $(1-5)$ one sees that $M(P(\mathbf{z}))$ is in fact a limit point of $L$ if and only if

$$
\begin{aligned}
M\left(P\left(x^{r_{1}}, \ldots, x^{r_{n}}\right)\right) & \neq M(P(\mathbf{z})) \\
& \text { for infinitely many } \mathbf{r} \in \mathbb{Z}^{n}
\end{aligned}
$$

It seems rather difficult to establish (1-6) for any particular polynomial, but there is good reason to believe that it can fail only for trivial reasons if $P(\mathbf{z})$ is an irreducible polynomial in more than one variable. So, in spite of the fact that $(1-6)$ has been proved for only a few of the polynomials presented here, we refer to them as "limit points" of Mahler's measure. We add, however, that the proof of the special case of $(1-5)$,

$$
\lim _{n \rightarrow \infty} M\left(P\left(x, x^{n}\right)\right)=M(P(x, y)),
$$

given in [Boyd 81b] is capable of yielding an explicit error term, but only the case of $P(x, y)=1+x+y$ has been worked out in detail. In this case,

$$
m\left(1+x+x^{n}\right)=m(1+x+y)+c(n) / n^{2}+O\left(n^{-3}\right),
$$

where $c(n)=-\sqrt{3} \pi / 6$ if $n \equiv 2 \bmod 3$ and $c(n)=$ $\sqrt{3} \pi / 18$ if $n \equiv 0$ or $1 \bmod 3$. One could establish (1-6) rigorously for a particular two-variable polynomial by computing the error term in the same way, and for most of the examples presented here, we expect the error term in $(1-7)$ to have the form $c(n) n^{-3 / 2}+O\left(n^{-2}\right)$, where $c(n)$ is a nontrivial, almost periodic function of $n$ (cf. [Boyd 81b, page 466]). However, in Section 6 we suggest a more algebraic approach that seems capable of establishing in some generality that the $M(P(x, y))$ listed here are indeed genuine limit points.

Section 2 describes several families of sparse reciprocal polynomials in two variables with small measure. Section 3 discusses some methods for computing measures of two-variable polynomials to high accuracy. Section 4 describes several methods we employed to search for small limit points of measures, and Section 5 summarizes our results. Section 6 proposes a technique for establishing that the measure of a two-variable polynomial is a true limit point of one-variable measures, and the last section discusses some known and conjectured explicit formulas for measures of multivariable polynomials.

\section{HEXANOMIALS WITH SMALL MEASURE}

A hexanomial is a polynomial composed of exactly six monomials; the height of a polynomial is its largest coefficient in absolute value. We describe several families of hexanomials in two variables having height 1 whose members have rather small measure.

In [Boyd 81b], it was suggested that $M(1+x+y)=$ $1.381356 \ldots$ is the smallest element of the second derived set of $\mathbb{L}$, i.e., the smallest limit point of limit points of measures. It was also conjectured there that this value is the smallest limit point of measures of nonreciprocal polynomials. It is easy to see that $M(1+x+y)$ is the measure of a reciprocal three-variable polynomial. Taking

$$
G(x, y, z)=x+y+z+x y+y z+z x
$$

and treating this as a linear polynomial in $z$, we see from (1-3) that

$$
\begin{aligned}
M(G) & =M(z(1+x+y)+(x+y+x y)) \\
& =M\left(\max \left(|1+x+y|,\left|1+x^{-1}+y^{-1}\right|\right)\right) \\
& =M(1+x+y),
\end{aligned}
$$

since $1+x^{-1}+y^{-1}$ is the complex conjugate of $1+x+y$ for $|x|=|y|=1$. The polynomial $G$ of $(2-1)$ is the generic reciprocal hexanomial of height 1 .

By specifying $z$ to be a power of $x$, one obtains from (1-5) sequences of polynomials in two variables whose measures converge to $M(G(x, y, z))$. For example, consider the following set of reciprocal polynomials: let $\varphi_{a}(x)$ denote the polynomial $\left(x^{a}-1\right) /(x-1)$, and write

$$
\begin{aligned}
& P_{a, b}(x, y)= \\
& \quad x^{\max (a-b, 0)}\left(\varphi_{a}(x)+\varphi_{b}(x) y+x^{b-a} \varphi_{a}(x) y^{2}\right) .
\end{aligned}
$$

It is not hard to prove that $P_{a, b}(x, y)$ is irreducible over $\mathbb{Q}$ if $\operatorname{gcd}(a, b)=1$. If $\operatorname{gcd}(a, b)=c$ with $a=a^{\prime} c$ and $b=b^{\prime} c$, then $P_{a, b}(x, y)=\varphi_{c}(x) P_{a^{\prime}, b^{\prime}}\left(x^{c}, y\right)$. So we need only consider $\operatorname{gcd}(a, b)=1$.

We compute

$$
\begin{aligned}
M\left(P_{a, b}\right) & =M\left(\left(x^{a}-1\right)+\left(x^{b}-1\right) y+\left(x^{b}-x^{b-a}\right) y^{2}\right) \\
& =M\left((x-1)+\left(x^{b}-1\right) y+\left(x^{b}-x^{b-1}\right) y^{2}\right) \\
& =M\left((x-1) y+\left(x^{b}-1\right)+x^{b}\left(1-x^{-1}\right) y^{-1}\right) \\
& \rightarrow M\left((x-1) y+(z-1)+z\left(1-x^{-1}\right) y^{-1}\right) \\
& =M\left(x y-y-1+z\left(1+y^{-1}-x^{-1} y^{-1}\right)\right) \\
& =M\left(x+y+1+z\left(1+y^{-1}+x^{-1}\right)\right) \\
& =M(1+x+y),
\end{aligned}
$$

where we have made several uses of $(1-4)$.

We write $M(a, b)$ for $M\left(P_{a, b}\right)$ in the following. The smallest two known measures of polynomials in at least 
two variables are given by $M(2,3)=1.255433 \ldots$ and $M(1,3)=M(2,1)=1.285734 \ldots$. Using methods described in the next section, we compute the values of $M(a, b)$ for $1 \leq a \leq 15$ and $1 \leq b \leq b^{*}(a)$, where $b^{*}(a)$ is approximately $3 a$. From this it appears that, for fixed $a$,

$$
\min _{b} M(a, b)=M(a, 2 a-1) .
$$

These data also suggest that $M(a, 2 a-1)$ is increasing in $a$. By $(1-5), M(a, 2 a-1) \rightarrow M(1+x+y)=1.381356 \ldots$ as $a \rightarrow \infty$. The numerical data for $a \leq 100$ suggest that the difference $m(1+x+y)-m(a, 2 a-1) \approx c n^{-13 / 8}$, although $c n^{-3 / 2}$ would seem more plausible. For example, the smallest values of $a$ with $M(a, 2 a-1)>1.37$ and $M(a, 2 a-1)>1.38$ are respectively, $M(9,17)=$ $1.370142 \ldots$ and $M(34,67)=1.380050 \ldots$. Since we wish to concentrate on $M(P(x, y))<1.37$, only $(a, b)$ with $1 \leq a \leq 8$ should appear on our list, assuming (2-4) is correct.

We also consider the similar families

$$
\begin{aligned}
& Q_{a, b}(x, y)= \\
& \quad x^{\max (a-b, 0)}\left(1+x^{a}+\left(1+x^{b}\right) y+x^{b-a}\left(1+x^{a}\right) y^{2}\right),
\end{aligned}
$$

with $a+b$ odd, and

$$
\begin{aligned}
& R_{a, b}(x, y)= \\
& \quad x^{\max (a-b, 0)}\left(1+x^{a}+\left(1-x^{b}\right) y-x^{b-a}\left(1+x^{a}\right) y^{2}\right),
\end{aligned}
$$

where $a$ and $b$ are both odd. The parity restrictions are imposed to avoid considering essentially the same polynomial more than once. In both cases, we require $\operatorname{gcd}(a, b)=1$. Using a computation similar to $(2-3)$, we observe that $M\left(Q_{a, b}\right) \rightarrow M(G)$ and $M\left(R_{a, b}\right) \rightarrow M(G)$.

Another family of small measure hexanomials that we study is

$$
S_{a, b, \epsilon}(x, y)=1+\left(x^{a}+\epsilon\right)\left(x^{b}+\epsilon\right) y+x^{a+b} y^{2},
$$

where $1 \leq a<b, \operatorname{gcd}(a, b)=1$, and $\epsilon= \pm 1$. If $a$ and $b$ are both odd then $S_{a, b,-}(-x,-y)=S_{a, b,+}(x, y)$, so we need only take $\epsilon=+1$ in this case. From (1$5)$, we have $M\left(S_{a, b, \epsilon}\right) \rightarrow M\left(1+(x+1)(y+1) z+x y z^{2}\right)$ as $b \rightarrow \infty$. We leave it as an exercise to verify that $M\left(1+(x+1)(y+1) z+x y z^{2}\right)=M(G)$.

The last family of hexanomials of interest are those of the form $T(x, y)=y f(x)+f^{*}(x)$. Clearly, $M(T(x, y))=$ $M(f(x))$, and we need only consider nonreciprocal polynomials $f(x)$. Experimentally, the smallest known measures of nonreciprocal polynomials arise from trinomials of the form $1 \pm x^{m} \pm x^{n}$, or as factors of such trinomials. We may assume $\operatorname{gcd}(m, n)=1$, and in this case the trinomial is either irreducible or factors as a nonreciprocal irreducible polynomial and a cyclotomic factor of the form $1 \pm x+x^{2}$. Using (1-5) again, we see that the measures $M\left(1 \pm x^{m} \pm x^{n}\right)$ tend to $M(1+x+y)$ in the limit.

\section{COMPUTING MEASURES OF TWO-VARIABLE POLYNOMIALS}

In [Boyd 98b], an algorithm is given that, in principle, can effectively compute $M(P(\mathbf{z}))$ to arbitrary accuracy without integration. Although this method has some theoretical applications and can provide useful bounds for $M(P(\mathbf{z}))$, it is not a practical method for computing even moderately high-precision values of $M(P(\mathbf{z}))$. Instead, one uses Jensen's formula (1-2) to reduce the $n$ dimensional integral defining $m(P(\mathbf{z}))$ to a sum of $(n-1)$ dimensional integrals and computes these using standard numerical techniques.

Suppose $P(x, y)$ has degree $d$ in $y$. Let $y_{1}(x), \ldots, y_{d}(x)$ denote the $d$ solutions of $P(x, y)=0$, which may be chosen to be continuous, piecewise analytic functions of $x$. Write

$$
P(x, y)=a_{0}(x) \prod_{k=1}^{d}\left(y-y_{k}(x)\right)
$$

and use (1-2) in its logarithmic form to write

$$
\int_{0}^{1} \log |P(x, e(s))| d s=\left|a_{0}(x)\right|+\sum_{k=1}^{d} \log ^{+}\left|y_{k}(x)\right|,
$$

where, as usual, $\log ^{+}|u|:=\max (\log |u|, 0)$. Now, let $x=e(t)$ and integrate over $t$ to obtain

$$
m(P(x, y))=m\left(a_{0}(x)\right)+\sum_{k=1}^{d} \int_{0}^{1} \log ^{+}\left|y_{k}(e(t))\right| d t .
$$

The endpoints of the integrals in (3-1) occur at points for which $P(x, y)=0$ on the torus $\mathbb{T}^{2}$. For reciprocal $P(x, y)$ with real coefficients, these occur at values of $|x|=1$ that are roots of the polynomial $F(x)=\operatorname{disc}_{y} P(x, y)$. To see this, notice that if $P(e(t), y)=0$, then also $P(e(-t), \bar{y})=$ 0 , by complex conjugation, and then $P(e(t), 1 / \bar{y})=0$, since $P$ is reciprocal. An endpoint of an interval of integration is a value $t=\alpha$ such that $\left|y_{k}(e(t))\right|>1$ for $\alpha<t<\alpha+\epsilon$ or $\alpha-\epsilon<t<\alpha$ and $\lim _{t \rightarrow \alpha}\left|y_{k}(e(t))\right|=1$. But $1 \longdiv { y _ { k } ( e ( t ) ) }$ is a different root of $P(e(t), y)$ for which 
$\lim _{t \rightarrow \alpha}\left|1 \overline{y_{k}(e(t))}\right|=1$, hence $P(e(\alpha), y)=0$ has a double root and so $\operatorname{disc}_{y} P(e(\alpha), y)=0$.

We illustrate this in the case of the polynomials $P_{a, b}$ of $(2-2)$. These are quadratic in $y$, so we may use the quadratic formula to solve $P(e(t), y)=0$. The equation $P_{a, b}(e(t), y)=0$ simplifies to

$$
\begin{gathered}
e((b-a) t / 2) \sin (a \pi t) y^{2}+\sin (b \pi t) y \\
\quad+e(-(b-a) t / 2) \sin (a \pi t)=0 .
\end{gathered}
$$

Hence the roots of (3-2) are $y_{1}$ and $y_{2}=1 / \overline{y_{1}}$, where $\left|y_{1}\right| \geq\left|y_{2}\right|$, so we may write $\left|y_{1}\right|=|f(a, b, t)| / g(a, t)$, with $g(a, t)=\left|x^{a}-1\right|=2|\sin (a \pi t)|$ and

$$
f(a, b, t)=|\sin (b \pi t)|+\sqrt{\sin ^{2}(b \pi t)-4 \sin ^{2}(a \pi t)} .
$$

If $|\sin (b \pi t)|<2|\sin (a \pi t)|$, then $f(a, b, t)$ is complex and $|f(a, b, t)|=2|\sin (a \pi t)|=g(a, t)$, so $\left|y_{1}\right|=1=\left|y_{2}\right|$; while if $|\sin (b \pi t)|>2|\sin (a \pi t)|$, then $f(a, b, t)$ is real and $f(a, b, t)>g(a, t)$, so that $\left|y_{1}\right|>1>\left|y_{2}\right|$. Since the leading term $a_{0}(x)$ is the product of cyclotomic polynomials $\varphi_{a}(x)$, Equation $(3-1)$ thus reduces to

$$
m\left(P_{a, b}\right)=\int_{0}^{1} \log ^{+}\left|y_{1}\right| d t=2 \int_{0}^{1 / 2} \log ^{+}\left|y_{1}\right| d t
$$

by symmetry. Let $\left(\alpha_{k}, \beta_{k}\right)$ denote the subintervals of $(0,1 / 2)$ for which $|\sin (b \pi t)|>2|\sin (a \pi t)|$. Then

$$
\begin{aligned}
m\left(P_{a, b}\right)= & 2 \sum_{k} \int_{\alpha_{k}}^{\beta_{k}} \log |f(a, b, t)| d t \\
& -2 \sum_{k} \int_{\alpha_{k}}^{\beta_{k}} \log |g(a, t)| d t .
\end{aligned}
$$

The integrals involving $\log g(a, t)$ are standard Clausen integrals, easily evaluated since they are values of the Bloch-Wigner dilogarithm $\mathcal{D}(x)$, which has a rapidly convergent series expansion. The function $\mathcal{D}(x)$ is denoted by polylog $(2, x, 1)$ in PARI/GP [Batut et al. 00]. In terms of $\mathcal{D}(x)$, we have

$$
\begin{aligned}
\int_{\alpha}^{\beta} \log |2 \sin (a \pi t)| d t & =\int_{2 \pi \alpha}^{2 \pi \beta} \log \left|x^{a}-1\right| \frac{d x}{i x} \\
& =\left[-\frac{\mathcal{D}\left(x^{a}\right)}{2 \pi a}\right]_{e(\alpha)}^{e(\beta)} .
\end{aligned}
$$

The integrals involving $\log |f(a, b, t)|$ are proper integrals unless $f(a, b, t)=0$. But since we assume $\operatorname{gcd}(a, b)=1$ and $|\sin (b \pi t)| \geq 2|\sin (a \pi t)|$, this can only occur at $t=0$.
In this case, we write

$$
\begin{aligned}
\int_{0}^{\beta_{1}}(\log |f(a, b, t)|-\log |g(a, t)|) d t= \\
\int_{0}^{\beta_{1}} \log (|f(a, b, t) / g(a, t)|) d t,
\end{aligned}
$$

and the latter integral has a finite limit at $t=0$. As discussed above $e\left(\alpha_{k}\right)$ and $e\left(\beta_{k}\right)$ are multiple roots of $P(e(t), y)=0$ and hence the graph of $f(a, b, t)$ has a vertical tangent at the endpoints of the intervals of integration. This is easily handled by splitting the interval in the middle and then using the change of variable $t \rightarrow \alpha_{k}+t^{2}$ and $t \rightarrow \beta_{k}-t^{2}$ at the endpoints. Then the standard Romberg integration routine intnum of PARI/GP easily computes the integrals to any desired accuracy.

Most of the polynomials $P(x, y)$ that we study here are quadratic in one variable, and their measures may be evaluated using a similar method. In this case, the two roots $y_{1}(t)$ and $y_{2}(t)$ of $P(e(t), y)$ are given by $\left(r(t) \pm \sqrt{r(t)^{2}-s(t)^{2}}\right) / s(t)$, where $r(t)$ and $s(t)$ are trigonometric polynomials. We integrate one of the $\pm y_{k}(e(t))$ over each interval $[\alpha, \beta]$ where the discriminant is positive. Often the function $s(t)$ is zero at a point $\gamma$ in an interval of integration, but integrating by parts allows one to remove the singularity:

$$
\begin{aligned}
& \int_{\alpha}^{\beta} \log |s(t)| d t= \\
& \quad(\beta-\gamma) \log |s(\beta)|+(\gamma-\alpha) \log |s(\alpha)| \\
& \quad+\int_{\alpha}^{\beta}(\gamma-t) \frac{s^{\prime}(t)}{s(t)} d t .
\end{aligned}
$$

Some polynomials that we study have $\operatorname{deg}_{y}(P(x, y))=$ 3 or 4 . While the cubic formula may be used in a similar way to compute measures when the degree is 3 , we find it simpler to use numerical methods. All the polynomials with degree 3 or 4 in $y$ that we study have the property that at most one branch has $\left|y_{k}(e(t))\right|>1$ for any $t$, so a polynomial root finder and a numerical integration routine (polroot and intnum in PARI/GP) suffice to compute the required integrals. In general, however, when the degree is large one expects many values of $k$ with $\left|y_{k}(e(t))\right|>1$, and then one must isolate each continuous branch. In this case, one combines the numerical solution of $P(e(t), y)=0$ with the numerical integration, in order to treat each branch $y_{k}(e(t))$ individually. This has been used in the studies described in [Boyd 02], but since it is not needed here we do not go into more details. 


\section{SEARCHING FOR SMALL MEASURES}

We describe three principal methods used to search for polynomials in two variables with small measure.

\subsection{Patterns in Coefficients}

The first few limit points were found by recognizing patterns in the lists of small measures of polynomials with small degree [Boyd 80, Boyd 89, Mossinghoff 98, Mossinghoff 04]. In fact, the smallest two limit points are already apparent in the list of Salem numbers less than 1.3. For example, $x^{10}-x^{6}-x^{5}-x^{4}+1, x^{10}-x^{7}-$ $x^{5}-x^{3}+1$, and $x^{8}-x^{5}-x^{4}-x^{3}+1$ are all minimal polynomials of small Salem numbers, suggesting the family $P_{1,3}\left( \pm x^{m}, \pm x^{n}\right)$ and thus the small measure $M\left(P_{1,3}(x, y)\right)$. After finding $P_{1,3}$ and $P_{2,3}$ in this way, the generalization to $P_{a, b}$ is natural, and thus the discovery that all of these have relatively small measures. The third smallest known limit point,

$$
(1+x)+\left(1-x^{2}+x^{4}\right) y+\left(x^{3}+x^{4}\right) y^{2},
$$

was discovered in a similar way in [Mossinghoff 98], after observing a number of polynomials with small measure having the form

$$
x^{4 n}+x^{3 n-1}+x^{2 n+3}-x^{2 n+2}+x^{2 n+1}+x^{n+1}+1 .
$$

These earlier calculations also produced a number of single-variable polynomials with small measure without obvious patterns in their coefficients. One might hope that multiplying one of these sporadic polynomials by a product of cyclotomic polynomials would reveal a sparse polynomial that indicates a limit point. A systematic method of searching for appropriate multiples uses the LLL lattice reduction algorithm. For example, beginning with the polynomial from [Mossinghoff 04],

$$
\begin{aligned}
f(x)=x^{44} & -x^{42}+x^{35}-x^{33}+x^{31}-x^{29}+x^{26} \\
& -x^{24}+x^{22}-x^{20}+x^{18}-x^{15}+x^{13} \\
& -x^{11}+x^{9}-x^{2}+1
\end{aligned}
$$

which has $M(f)=1.291273 \ldots$, consider the lattice spanned by $f(x) g_{k}(x)$, where $g_{0}(x)=x^{m}$ and $g_{k}(x)=$ $x^{m+k}+x^{m-k}$ for $1 \leq k \leq m$. Employing LLL to find a small vector in this lattice, when $m=4$ one detects the sparse multiple

$$
x^{52}+x^{51}+x^{39}+x^{38}+x^{26}+x^{14}+x^{13}+x+1,
$$

which, for $y=x^{13}$, is

$F(x, y)=y^{4}+y^{4} x^{-1}+y^{3}+y^{3} x^{-1}+y^{2}+x y+y+x+1$.
In this case, $x F\left(x^{-1}, y\right)=P_{2,5}(y, x)$ and $M\left(P_{2,5}\right)=$ $1.332051 \ldots$, but this is certainly not apparent from $(4-2)$.

\subsection{Clusters of Measures}

A second method of searching for small limit points of Mahler's measure involves computing the measures of a large number of single-variable polynomials of a particular form. We first consider primitive reciprocal hexanomials of height 1: polynomials with the form $1+r x^{a}+s x^{b}+s x^{d-b}+r x^{d-a}+x^{d}$, with $r$ and $s$ equal to $\pm 1,0<a<b<d / 2$, and $\operatorname{gcd}(a, b)=1$. Computing the measure of all 454,368 of these with $d \leq 150$ and counting the number in each interval of the form $((k-1 / 2) / 1000,(k+1 / 2) / 1000]$, we obtain the histogram shown in Figure 1. We also consider heptanomials, or seven-term polynomials. The histogram for the measures of the 456,968 primitive reciprocal heptanomials with height 1 and $d \leq 150$ is displayed in Figure 2 .

It is apparent that these distributions are not uniform, and one can distinguish many peaks of various widths in both graphs. By examining the list of polynomials with measures in the neighbourhood of each peak, one can usually find a pattern that identifies a limit point $P(x, y)$. For example, our two smallest measures, 1.2554... and $1.2857 \ldots$, are readily apparent in both graphs. Their appearance in Figure 1 is clear from the discussion in Section 2; their appearance in Figure 2 stems from the fact that $P_{2,3}(x, y)$ and $\left(1-x+x^{2}\right) P_{2,1}(x, y)$ are heptanomials of height 1 . As we note in Section 6, the sharp spikes in Figure 1 are due to the geometric convergence

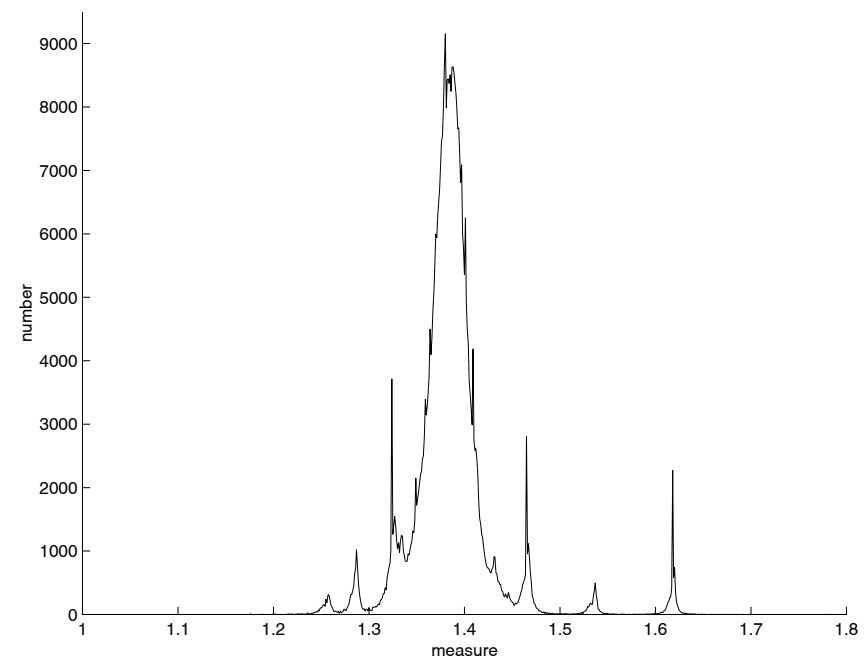

FIGURE 1. Reciprocal hexanomials: height 1 , degree $\leq 150$. 


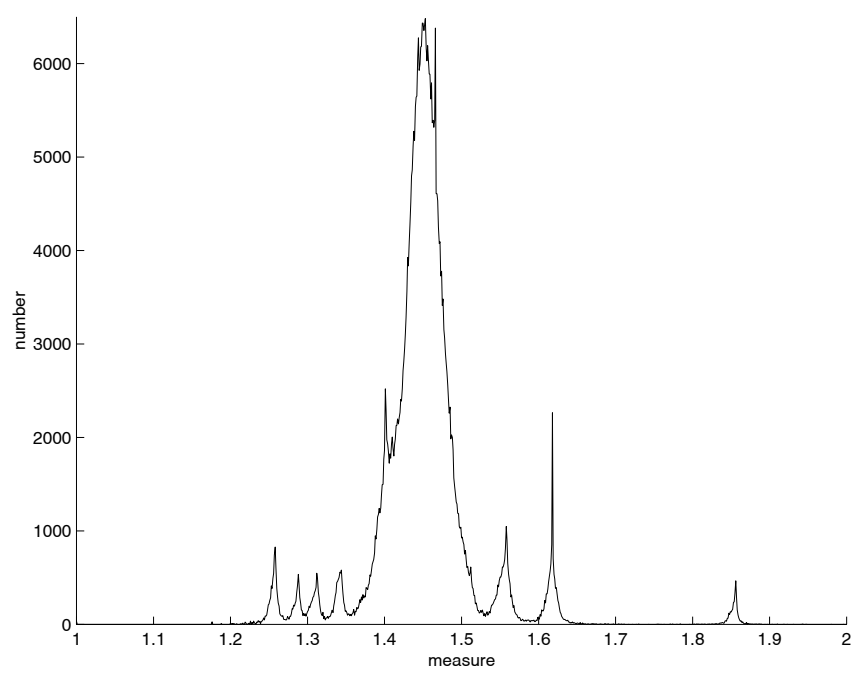

FIGURE 2. Reciprocal heptanomials: height 1 , degree $\leq 150$.

of $M\left(x^{n} f(x)+f^{*}(x)\right)$ to $M\left(y f(x)+f^{*}(x)\right)$. The spike arising from $f(x)=x^{3}-x-1$ with $M(f(x))=1.3247 \ldots$ is readily apparent in Figure 1. The sharp spikes in Figure 2 have a similar cause. For example, the spike near 1.465 in Figure 2 is due to the limit point $P(x, y)=(1+$ $\left.x+x^{3}\right)+x^{2} y+x\left(1+x^{2}+x^{3}\right) y^{2}$, which has $M(P(x, y))=$ $M\left(1+x+x^{3}\right)=1.4655712318767680267 \ldots$.

Each graph has one broad central peak corresponding to an element of the second derived set of $\mathbb{L}$ and a threevariable polynomial. The main peak in Figure 1 centers on the generic reciprocal hexanomial of height 1 ,

$$
M(x+y+z+1 / x+1 / y+1 / z)=
$$

$$
1.3813564445184977933 \ldots \text {; }
$$

that of Figure 2 lies on the generic reciprocal heptanomial of height 1 ,

$$
\begin{array}{r}
M(x+y+z+1+1 / x+1 / y+1 / z)= \\
1.4483035845491699038 \ldots .
\end{array}
$$

The first of these is relatively easy to compute accurately since it reduces to $M(1+x+y)$ and thence to a single integral by Jensen's formula (in fact, it is made even easier by an exact formula of Smyth that we mention in the final section). The second can be reduced to a double integral by Jensen's formula, but it would be time-consuming to attain the accuracy claimed in (4-3) by numerical integration. However, as Rodriguez Villegas remarks in [Rodriguez Villegas 99], the methods of that paper extend to certain families of K3 surfaces, and Bertin [Bertin 05] recently used these methods to produce a rapidly convergent series for $M(x+y+z+k+1 / x+1 / y+1 / z)$. This was used to compute (4-3).

We remark that the two largest limit points of measures of hexanomials appearing in Figure 1 are $M\left(Q_{1,2}\right)=1.535098 \ldots$, with $Q_{a, b}$ given by $(2-5)$, and the golden ratio. The two largest visible limit points for heptanomials in Figure 2 are the golden ratio (arising from $\left.1-x-x^{2}+x y-\left(1+x-x^{2}\right) y^{2}\right)$ and $M(1+x+$ $\left.\left(1-x+x^{2}\right) y+x(1+x) y^{2}\right)=1.853120 \ldots$.

\subsection{Systematic Searches}

We conducted several systematic searches for twovariable polynomials with small measure. We first computed the measures of several polynomials from each family of hexanomials described in Section 2.

- $P_{a, b}(x, y)$, for $1 \leq a \leq 15, \operatorname{gcd}(a, b)=1$, and $1 \leq b \leq b^{*}(a)$, where $b^{*}(a)$ is given by the list $[16,17,13,15,18,19,23,25,28,29,30,31,37,39,41]$.

- $Q_{a, b}(x, y)$, for $a \leq 20, b \leq 20, \operatorname{gcd}(a, b)=1$, and $a+b$ odd.

- $R_{a, b}(x, y)$, for $a \leq 19, b \leq 19, \operatorname{gcd}(a, b)=1$, and $a$ and $b$ both odd.

- $S_{a, b, \epsilon}(x, y)$, for $1 \leq a<b \leq 15, \operatorname{gcd}(a, b)=1$, and $\epsilon= \pm 1$.

- $T(x, y)=f(x)+y f^{*}(x)$, for trinomials $f(x)$ with height 1 and $\operatorname{deg}(f) \leq 50$.

We also searched two large families of two-variable polynomials.

- Reciprocal polynomials $P(x, y)$ with height 1 having $\operatorname{deg}_{x}(P) \leq 9$ and $\operatorname{deg}_{y}(P)=2$. These polynomials have the form $P(x, y)=f(x)+x^{a} g(x) y+x^{b} f^{*}(x) y^{2}$, with $g(x)$ reciprocal and $a$ and $b$ chosen so that $P$ is reciprocal. If $f(0)=0$, then $P(x, x y) / x$ is a reciprocal polynomial of height 1 with the same measure as $P$, so we may assume $f(0)=1$ without loss of generality. However, this operation increases $b$, so the normalized polynomial might lie outside our search space. Consequently, we enforced the weaker constraint that $P(x, 0) P(0, y) \neq 0$, together with some simple constraints to guarantee that none of $P(-x, y), P(x,-y),-P(x, y), y^{2} P\left(x, y^{-1}\right)$, or some combination of these, is constructed once $P(x, y)$ is checked. 
- Symmetric, reciprocal $P(x, y)$ with height 1 and $d \leq 6$, where $d=\operatorname{deg}_{x}(P)=\operatorname{deg}_{y}(P)$, and the same set for $d=7$ in the case that $P(x, 0)$ has at most two terms. The coefficients of these polynomials are symmetric about both diagonals, so there are $(d+1)^{2} / 4$ coefficients that may be set to 0,1 , or -1 for odd $d$ and $d(d+2) / 4$ such coefficients when $d$ is even. The number of possibilities is reduced somewhat by requiring that $P(x, 0) \neq 0$ and ensuring that we construct only one of $\{ \pm P(x, y), \pm P(-x,-y)\}$.

The numerical methods of Section 3 are impractical for testing the large number of candidate polynomials in these latter two categories. We require instead a simple procedure to determine if $M(P(x, y))<M_{0}$ for a particular bound $M_{0}$. In searches of single-variable polynomials [Boyd 80, Boyd 89, Mossinghoff 98], the method of root squaring, together with bounds on coefficients of polynomials having bounded measure, provides a simple and fast means of screening candidate polynomials. The multivariable version of this algorithm introduced in [Boyd 98b] could, in principle, be used in the same way. Fixing the support of a multivariable polynomial $P$ and supplying an upper bound $M_{0}$ on $M(P)$, Mahler's inequalities [Mahler 62], [Schinzel 00, page 247] provide bounds on the coefficients of $P$. For two-variable polynomials, the role of root squaring is supplied by the operator $\mathcal{T}$, defined by

$$
\begin{aligned}
\mathcal{T} P(x, y)= & P\left(x^{1 / 2}, y^{1 / 2}\right) \\
& \times P\left(-x^{1 / 2}, y^{1 / 2}\right) \\
& \times P\left(x^{1 / 2},-y^{1 / 2}\right) \\
& \times P\left(-x^{1 / 2},-y^{1 / 2}\right) .
\end{aligned}
$$

It is clear that $\mathcal{T} P(x, y)$ is a polynomial with $M(\mathcal{T} P)=$ $M(P)^{4}$. Thus, the coefficients of $\mathcal{T}^{k}(P)$ must satisfy Mahler's inequalities with the bound $M_{0}^{4^{k}}$. If $\ell_{1}(P)$ denotes the sum of the absolute values of the coefficients of $P$, then $\ell_{1}\left(\mathcal{T}^{k} P\right)^{1 / 4^{k}}$ approaches $M(P)$ as $k \rightarrow \infty$ [Boyd 98b]. While it appears that this could provide an effective method for detecting polynomials $P$ having $M(P)>M_{0}$, in fact, the support of $\mathcal{T}^{k} P$ grows exponentially with $k$, and this limits the utility of this method.

Instead, we use (1-7) for screening out unsuitable polynomials. Given a polynomial $P(x, y)$, we compute the mean of the single-variable measures $M\left(P\left(x, x^{n_{i}}\right)\right)$ for a particular set of positive integers $\left\{n_{1}, \ldots, n_{r}\right\}$ and discard $P(x, y)$ if this average exceeds a particular bound $B$. Of course, without an error bound in (1-7), this is only a heuristic method since one may make a particularly unlucky choice of the $n_{i}$, but it is reasonably ef- ficient. We iterate this procedure, gradually decreasing the bound $B$ while increasing the $n_{i}$. For example, we tested 480,068 polynomials $P(x, y)$ with height 1 having $\operatorname{deg}_{x}(P)=9$ and $\operatorname{deg}_{y}(P)=2$. All but 8,677 of these (or their irreducible factors) were removed in an initial screening using $B=1.65$, and only 928 survived a second round with $B=1.45$. Two more rounds with $B=1.40$ then $B=1.38$ leave 300 polynomials. Examining each of these reveals that 223 have measure less than 1.37, and that each one is equivalent to one of 36 polynomials in the list given in the next section (specifically, those ranked 1-22, 24-29, 33, 34, 36-38, and 44-46).

\section{LIMIT POINTS LESS THAN 1.37}

Our searches detected 48 irreducible polynomials in two variables having integer coefficients and Mahler's measure greater than 1 and less than 1.37. These are listed in Table 1. Thirty have the form $P_{a, b}(x, y)$ as in $(2-2)$; these are labeled as $P(a, b)$ in the table. One polynomial from each of the families $Q_{a, b}(x, y)$ and $R_{a, b}(x, y)$ in $(2-5)$ and (2-6) also appears; these are labeled in a similar way. Six are instances of $S_{a, b, \epsilon}(x, y)$ in $(2-7)$; these are marked as $S(a, b, \operatorname{sgn}(\epsilon))$. Five more are linear in $y$, having the form $T(x, y)=y f(x)+f^{*}(x)$ discussed in Section 2. These are denoted by $T(f(x))$, where $f$ is a trinomial that realizes the measure. If $f$ is reducible, the coefficients of its noncyclotomic part are also shown.

The remaining five polynomials are identified by displaying the rows of their coefficient matrix, using + and - as abbreviations for 1 and -1 (all have height 1). For example, the third smallest known limit point $(4-1)$ is identified by the sequence $[++000,+0-0+, 000++]$.

At least 44 of the measures in our list are realized by hexanomials: the 43 in the described families plus the bicubic ranked number 30. One can recognize many of these values as "spikes" in the histogram of Figure 1. The third limit point is evident in Figure 2, but is not apparent in Figure 1, so we can be fairly confident that our heptanomial (4-1) does not divide a hexanomial of height 1 with the same measure.

Occasionally in our searches we detect an irreducible polynomial of height 2 with small measure (when it occurs as a factor of a polynomial with height 1). The smallest measure we find here is $M\left(x^{3}+x(1+\right.$ $\left.x+x^{2}+x^{3}\right) y+x(x+1)^{2} y^{2}+\left(1+x+x^{2}+x^{3}\right) y^{3}+$ $\left.x y^{4}\right)=1.3724860771580461413 \ldots ;$ another interesting example is $M\left(\left(1+x+x^{2}\right)\left(1+y+y^{2}\right)+x y\right)=$ $1.3823754938807745701 \ldots$.

More detailed results of our searches are available in [Mossinghoff 04]. 
Measure

1. 1.2554338662666087457

2. $\quad 1.2857348642919862749$

3. $\quad 1.3090983806523284595$

4. 1.3156927029866410935

5. $\quad 1.3247179572447460260$

6. 1.3253724973075860349

7. $\quad 1.3320511054374193142$

8. 1.3323961294587154121

9. 1.3381374319388410775

10. $\quad 1.3399999217381835332$

11. 1.3405068829308471079

12. 1.3497161046696958653

13. 1.3500148321630142650

14. 1.3503169790598690950

15. 1.3511458956697046903

16. 1.3524680625188602961

17. 1.3536976494626355711

18. 1.3567481051456008311

19. 1.3567859884526454967

20. 1.3581296324044179208

21. 1.3585455903960511404

22. $\quad 1.3592080686995589268$

23. 1.3598117752819405021

24. 1.3598158989877492950

25. 1.3599141493821189216

26. 1.3602208408592842371

27. 1.3627242816569882815

28. 1.3636514981864992177

29. 1.3641995455827723418

30. 1.3644358117806362770

31. $\quad 1.3645459857899151366$

32. 1.3646557293930641449

33. 1.3650623157174417179

34. 1.3654687370557201592

35. 1.3659850533667936783

36. $\quad 1.3661459663116649518$

37. $\quad 1.3665709746056369455$

38. 1.3668078899273126149

39. 1.3668830708592258921

40. 1.3669909125179202255

41. $\quad 1.3677988580117157740$

42. 1.3678546316653002345

43. $\quad 1.3681962517212729703$

44. 1.3682140096679950123

45. 1.3683434385467330804

46. $\quad 1.3687474425069274154$

47. $\quad 1.3689491694959833864$

48. 1.3697823199880122791

TABLE 1. Known limit points less than 1.37.

\section{TRUE LIMIT POINTS}

Polynomial

$P(2,3)$

$P(1,3)$ or $P(2,1)$

$[++000,+0-0+, 000++]$

$P(3,5)$

$T\left(1+x-x^{3}\right)$

$P(3,4)$

$P(2,5)$

$S(1,3,+)$

$P(3,2)$

$P(4,7)$

$P(3,1)$

$T\left(1+x^{2}-x^{7}\right)[+++0--]$

$P(3,7)$

$S(1,4,-)$

$P(4,5)$

$P(5,9)$

$Q(1,6)$

$P(4,3)$

$P(5,8)$

$[++00000,+0---0+$, $00000++]$

$P(4,1)$

$P(4,9)$

$P(6,11)$

$S(1,6,+)$

$T\left(1+x+x^{8}\right) \quad[+0-+0-+]$

$P(5,7)$

$P(5,6)$

$S(3,5,+)$

$T\left(1-x^{2}+x^{5}\right)$

$[+000,00++,++00,000+]$

$P(7,13)$

$P(5,11)$

$S(2,7,-)$

$P(5,4)$

$[++000,++0-0,00000$, $0-0++, 000++]$

$P(5,3)$

$P(5,2)$

$P(5,1)$

$R(1,5)$

$P(7,12)$

$P(8,15)$

$T\left(1+x^{4}+x^{11}\right)$

$[+-0+0-+0-+]$

$P(6,13)$

$P(1,9)$

$[++00000,++0-0++$ $00000++]$

$P(6,7)$

$P(7,11)$

$S(1,9,+)$
We suggest a possible method of proving that $M(P(x, y))$ is a limit point of one-dimensional measures by showing that $M\left(P\left(x, x^{n}\right)\right) \neq M(P(x, y))$ for all sufficiently large $n$. We begin by discussing the distribution of the zeros of $P\left(x, x^{n}\right)$. For simplicity, we first consider a specific example, $P=P_{2,1}$. For each $x$ let $y_{k}(x)$, with $k=1$ or 2 , denote the zeros of $P(x, y)=0$. If $R(x)$ denotes the polynomial $\operatorname{disc}_{y} P(x, y)=x^{4}-2 x^{3}-5 x^{2}-2 x+1$, then $R$ has two zeros on the unit circle, $\xi$ and $\bar{\xi}$, with $\alpha=\arg \xi=2.724359 \ldots$. Using the methods of Section 3, we find that $\left|y_{1}(e(t))\right|>1$ for $\alpha<|t| \leq 1 / 2$ and $\left|y_{1}(e(t))\right|=1$ for $|t| \leq \alpha$. Since $P\left(x, x^{n}\right)$ is a sparse polynomial of fixed length, an elementary analysis shows that the arguments of its zeros are uniformly distributed around the unit circle. Further, since $P\left(x, x^{n}\right)=0$ is equivalent to $y_{k}(x)=x^{n}$ for $k=1$ or 2 , it is not hard to see that for a zero of the form $x=\rho(t) e^{i t}$, one has asymptotically $\rho(t) \approx\left|y_{k}(e(t))\right|^{1 / n}$ for $k=1$ or 2 . Thus the number of zeros of $P\left(x, x^{n}\right)$ outside the unit circle is asymptotic to $\delta \operatorname{deg}\left(P\left(x, x^{n}\right)\right)$, where $\delta=(\pi-\alpha) /(2 \pi)=$ $.06640475 \ldots$. Note that $\operatorname{deg}\left(P\left(x, x^{n}\right)\right)=2 n$.

In general, let $P(x, y)$ be of degree $d$ in $y$, let $\nu(x)$ denote the number of roots of $P(x, y)=0$ with $|y(x)|>1$. Define $\delta=\delta(P):=\int_{0}^{1} \nu(e(t)) d t$. Then $0 \leq \delta \leq 1(\delta \leq$ $1 / 2$ if $P$ is reciprocal). If $0<\delta<1$ then the number of zeros of $P\left(x, x^{n}\right)$ outside the unit circle is asymptotically $\sim \delta d n$. For example, $\delta(1+x+y)=1 / 3$ and $\delta\left(P_{1,3}\right)=1 / 6$, but usually, as with $\delta\left(P_{2,1}\right)=.06640475 \ldots$, the value of $\delta(P)$ is irrational. It is possible that $\delta=0$, as for $P(x, y)=y\left(x^{3}-x-1\right)+\left(x^{3}+x^{2}-1\right)$ or $P(x, y)=$ $\left(x^{4}+x+1\right) y^{2}+x^{2} y+\left(x^{4}+x^{3}+1\right)$. Similarly, $\delta=1$ is possible, as for $P=x+y+3$. The argument described in the next paragraph assumes that $\{P(x, y)=0\}$ has a nontrivial intersection with the torus, by which we mean that $0<\delta(P)<1$.

Notice that $\beta= \pm M\left(P\left(x, x^{n}\right)\right)$ is the product of the zeros of $P\left(x, x^{n}\right)$ outside the unit circle and hence is an algebraic integer. Since $P\left(x, x^{n}\right)$ has degree $d n$ and has about $\delta d n$ zeros outside the unit circle, formally, $\beta$ has roughly $\left(\begin{array}{c}d n \\ \lfloor\delta d n\rfloor\end{array}\right)$ conjugates, and this number tends to infinity exponentially with $n$. On the other hand, if $M\left(P\left(x, x^{n}\right)\right)=M(P(x, y))$ for all large $n$, then $\beta$ has a fixed set of Galois conjugates independent of $n$. For this to occur, the Galois group of $P\left(x, x^{n}\right)$ would have to be highly unusual. Even granted that $P\left(x, x^{n}\right)$ may be reducible and, furthermore, is a reciprocal polynomial so its Galois group is nongeneric, it seems highly implausible that the Galois orbit of $\beta$ can be restricted in this 
way. It would be interesting to see this argument made rigorous even for a single example such as $P_{1,3}$ or $P_{2,3}$.

The above argument does not apply to $P(x, y)=$ $y f(x)+f^{*}(x)$ with $f(x)$ nonreciprocal. In this case it is easy to see by Rouché's theorem that for sufficiently large $n, P\left(x, x^{n}\right)$ has the same number of zeros outside $|x|=1$ as $f(x)$ does. Then $M\left(P\left(x, x^{n}\right)\right)$ approaches $M(P(x, y))=M(f(x))$ geometrically, in fact, like $O\left(\rho^{n}\right)$ where $1 / \rho$ is the minimum of $|\alpha|$ for those roots of $f(x)$ with $|\alpha|>1$. This geometric convergence explains why the "spikes" in Figure 1 corresponding to these $M(P(x, y))$ are so sharp, compared with the broad peaks corresponding to the $M\left(P_{a, b}(x, y)\right)$, for example. It is also easy to establish for these $P(x, y)$ that $M\left(P\left(x, x^{n}\right)\right) \neq M(P(x, y))$ for all $n$, so that $M(P(x, y))$ is a true limit point. For example, if $f(x)=x^{3}-x-1$, the minimal polynomial of the smallest Pisot number $\theta_{0}$, then $P\left(x, x^{n}\right)$ defines a sequence of Salem numbers $\theta_{n}$ with $\left|\theta_{n}-\theta_{0}\right| \sim 1 /\left(\left|f^{\prime}\left(\theta_{0}\right)\right| \theta_{0}^{n}\right)$. This is a well-known result of Salem [Salem 45].

\section{EXPLICIT FORMULAS}

Explicit formulas are known for some of the $M(P(x, y))$ appearing in the list in Section 5. These are more naturally expressed in terms of the logarithmic Mahler's measure $m(P(x, y))$. The earliest of these was Smyth's formula [Boyd 81b],

$$
m(1+x+y)=\frac{3 \sqrt{3}}{4 \pi} L\left(2, \chi_{-3}\right)=L^{\prime}\left(-1, \chi_{-3}\right)
$$

where $L\left(s, \chi_{-3}\right)$ denotes the Dirichlet $L$-series for the character $\chi_{-3}(n)=\left(\frac{-3}{n}\right)$, i.e., $\sum_{n=1}^{\infty} \chi_{-3}(n) n^{-s}$.

In [Boyd and Rodriguez Villegas 02], a generalization of Smyth's formula is given for polynomials of the form $P(x, y)=y f(x)+g(x)$, where $f(x)$ and $g(x)$ are cyclotomic polynomials. In many cases, these formulas reduce to a single term of the form $r L^{\prime}\left(-1, \chi_{-d}\right)$, where $r$ is a rational number. However, none of these values of $m(y f(x)+g(x))$ is small enough to appear on our list.

There are also conjectured formulas for $m(P(x, y))$ for many polynomials that define algebraic curves of genus 1 or 2. Some of these do appear in our list; for example, the two smallest known limit points appear to satisfy

$$
m\left(1+x+\left(1+x+x^{2}\right) y+\left(x+x^{2}\right) y^{2}\right) \stackrel{?}{=} L^{\prime}(14 A, 0)
$$

and

$$
m\left(1+\left(1+x+x^{2}\right) y+x^{2} y^{2}\right) \stackrel{?}{=} L^{\prime}(15 A, 0) .
$$

Here, $L(E, s)$ denotes the $L$-function for an elliptic curve $E$, and $14 A$ and $15 A$ denote (isogeny classes of) elliptic curves of conductors 14 and 15, respectively. The symbol $\stackrel{?}{=}$ indicates that the identity has been verified to at least 28 decimal places, but has not yet been proved.

Formula (7-3) was conjectured by Deninger [Deninger 97], and this led to the infinitely many conjectures of [Boyd 98a], including (7-2). Other polynomials in our list for which formulas are conjectured are

$$
\begin{gathered}
m\left(P_{3,4}\right) \stackrel{?}{=}-L^{\prime}(185 B, 0) / 12, \\
m\left(P_{3,2}\right) \stackrel{?}{=} L^{\prime}(34 A, 0) / 3 \\
m\left(P_{3,1}\right) \stackrel{?}{=} L^{\prime}(105 A, 0) / 12 \\
m\left(P_{1,4}\right) \stackrel{?}{=}-L^{\prime}(57 A, 0) / 2, \text { and } \\
m\left(\left(1+x+x^{2}\right)\left(1+y+y^{2}\right)+x y\right) \stackrel{?}{=} L^{\prime}(40 A) / 3
\end{gathered}
$$

The last polynomial is one of the examples we cited with height 2 .

It is worth remarking that it was the existence of a list of accurately computed values of $m\left(P_{a, b}\right)$ that led to the experiments of [Boyd 98a] following Deninger's remarkable prediction (7-3).

Of course, we also have explicit formulas for the examples $m\left(y f(x)+f^{*}(x)\right)$, but these are of a more elementary nature. There are other elementary examples in which $m\left(f(x) y^{2}+g(x) y+f^{*}(x)\right)=m(f(x))$, for example, when $f(x)=1+x+x^{4}$ and $g(x)=x^{2}$, and similar examples of higher degree in $y$.

All the other polynomials on our list with $\operatorname{deg}_{y}(P)>1$ define curves of genus greater than 2 and, so far, we do not have even conjectured formulas for these. The main theorem of [Deninger 97] assumes that $P(x, y)$ does not vanish on the torus, so it does not apply to any of the polynomials on our list. However, the ideas in [Deninger 97] would suggest that there may be explicit formulas even for these higher genus cases.

There are also formulas known (in fact, infinitely many) for the measure of the so-called $A$-polynomial (n.b., not the Alexander polynomial) $A(x, y)$ of a hyperbolic manifold [Boyd 02]. In such cases $A(x, y)$ may define a curve of large genus, but $m(A(x, y))$ is expressible in terms of dilogarithms so is more akin to the formula $(7-1)$. These formulas are explained by the way the curve $\mathcal{Z}=\{A(x, y)=0\}$ intersects the torus $\mathbb{T}^{2}$ rather than by the cohomology of the curve $\mathcal{Z}$. None of these examples seems to have particularly small Mahler's measure and, hence, these do not appear in this paper. 
On the other hand, Silver and Williams [Silver and Williams 04] have shown that many of our small limit points occur as measures of Alexander polynomials of two-component links. For example, the first two numbers in our list are the measures of the Alexander polynomials of the links $7_{1}^{2}$ and $6_{2}^{2}$, respectively. They show that the third smallest known limit point also arises as Mahler's measure of the Alexander polynomial of a link.

\section{ACKNOWLEDGMENTS}

This research was supported by a grant from NSERC.

\section{REFERENCES}

[Batut et al. 00] C. Batut, K. Belabas, D. Bernardi, H. Cohen, and M. Olivier. "User's Guide to PARI/GP." Available from World Wide Web (http://pari.math .u-bordeaux.fr), 2000 .

[Bertin 05] M. J. Bertin. "Mesure de Mahler d'hypersurfaces K3." 2005. ArXiv:math.NT/0501153.

[Boyd 77] D. W. Boyd. "Small Salem Numbers." Duke Math. J. 44:2 (1977), 315-328.

[Boyd 80] D. W. Boyd. "Reciprocal Polynomials Having Small Measure." Math. Comp. 35:152 (1980), 1361-1377.

[Boyd 81a] D. W. Boyd. "Kronecker's Theorem and Lehmer's Problem for Polynomials in Several Variables." J. Number Theory 13:1 (1981), 116-121.

[Boyd 81b] D. W. Boyd. "Speculations Concerning the Range of Mahler's Measure." Canad. Math. Bull. 24:4 (1981), 453-469.

[Boyd 89] D. W. Boyd. "Reciprocal Polynomials Having Small Measure. II." Math. Comp. 53:187 (1989), 355357, S1-S5.

[Boyd 98a] D. W. Boyd. "Mahler's Measure and Special Values of L-Functions." Experiment. Math. 7:1 (1998), 3782 .

[Boyd 98b] D. W. Boyd. "Uniform Approximation to Mahler's Measure in Several Variables." Canad. Math. Bull. 41:1 (1998), 125-128.

[Boyd 02] D. W. Boyd. "Mahler's Measure and Invariants of Hyperbolic Manifolds." In Number Theory for the Millennium, I (Urbana, IL, 2000), pp. 127-143. Natick, MA: A K Peters, 2002.

[Boyd and Rodriguez Villegas 02] D. W. Boyd and F. Rodriguez Villegas. "Mahler's Measure and the Dilogarithm. I." Canad. J. Math. 54:3 (2002), 468-492.

[Breusch 51] R. Breusch. "On the Distribution of the Roots of a Polynomial with Integral Coefficients." Proc. Amer. Math. Soc. 2:6 (1951), 939-941.
[Deninger 97] C. Deninger. "Deligne Periods of Mixed Motives, $K$-Theory and the Entropy of Certain $\mathbf{Z}^{n}$ Actions." J. Amer. Math. Soc. 10:2 (1997), 259-281.

[Flammang et al. 06] V. Flammang, G. Rhin, and J. -M. SacÉpée. "Integer Transfinite Diameter and Polynomials with Small Mahler Measure." To appear in Math. Comp., 2006.

[Lawton 83] W. M. Lawton. "A Problem of Boyd Concerning Geometric Means of Polynomials." J. Number Theory 16:3 (1983), 356-362.

[Lehmer 33] D. H. Lehmer. "Factorization of Certain Cyclotomic Functions." Ann. of Math. (2) 34:3 (1933), 461479 .

[Mahler 62] K. Mahler. "On Some Inequalities for Polynomials in Several Variables." J. London Math. Soc. 37 (1962), 341-344.

[Mossinghoff 98] M. J. Mossinghoff. "Polynomials with Small Mahler Measure." Math. Comp. 67:224 (1998), 16971705, S11-S14.

[Mossinghoff 04] M. J. Mossinghoff. "Lehmer's Problem." Available from World Wide Web (http://www.cecm.sfu .ca/ mjm/Lehmer), 2004.

[Mossinghoff et al. 98] M. J. Mossinghoff, C. G. Pinner, and J. D. Vaaler. "Perturbing Polynomials with All Their Roots on the Unit Circle." Math. Comp. 67:224 (1998), 1707-1726.

[Rhin and Sac-Épée 03] G. Rhin and J. -M. Sac-Épée. "New Methods Providing High Degree Polynomials with Small Mahler Measure." Experiment. Math. 12:4 (2003), 457461.

[Rodriguez Villegas 99] F. Rodriguez Villegas. "Modular Mahler Measures. I." In Topics in Number Theory (University Park, PA, 1997), pp. 17-48, Math. Appl. 467. Dordrecht: Kluwer Acad. Publ., 1999.

[Salem 45] R. Salem. "Power Series with Integral Coefficients." Duke Math. J. 12 (1945), 153-172.

[Schinzel 00] A. Schinzel. Polynomials with Special Regard to Reducibility, Encyclopedia Math. Appl., 77. Cambridge, UK: Cambridge Univ. Press, 2000.

[Siegel 44] C. L. Siegel. "Algebraic Integers whose Conjugates Lie in the Unit Circle." Duke Math. J. 11 (1944), 597602.

[Silver and Williams 04] D. S. Silver and S. G. Williams. "Mahler Measure of Alexander Polynomials." J. London Math. Soc. (2) 69:3 (2004), 767-782.

[Smyth 71] C. J. Smyth. "On the Product of the Conjugates Outside the Unit Circle of an Algebraic Integer." Bull. London Math. Soc. 3 (1971), 169-175. 
414 Experimental Mathematics, Vol. 14 (2005), No. 4

David W. Boyd, Department of Mathematics, University of British Columbia, Vancouver, B.C. V6T 1Z2, Canada (boyd@math.ubc.ca)

Michael J. Mossinghoff, Department of Mathematics, Davidson College, Davidson, North Carolina 28035-6996 (mjm@member.ams.org)

Received January 20, 2005; accepted June 14, 2005. 